\title{
Desastres naturales y territorialidad: el caso de los lafkenche de Saavedra'
}

\author{
Francisco Molina Camacho², Jorge Constanzo Belmar³ \\ y Cristián Inostroza Matus ${ }^{4}$
}

\begin{abstract}
RESUMEN
Los desastres naturales son característicos en Chile, terremotos, tsunamis, erupciones volcánicas e inundaciones son fenómenos relativamente frecuentes, cuya acción, muchas veces altera la forma como los grupos humanos se relacionan con la naturaleza. Esto, debido a su capacidad de modificar prácticas culturales, sociales y económicas, reorientando así, el devenir de un determinado territorio, tal como sucedió con el terremoto $9,5^{\circ}$ Richter y posterior tsunami de 1960 , en la comuna de Saavedra, Chile. Desde una ecología política de los desastres; y a través de grupos focales, entrevistas semi-estructuradas y mapeos participativos; el presente artículo analiza la forma como las autoridades locales con competencia en materia de desastres han reconocido la importancia de la territorialidad del pueblo lafkenche, generándose un dialogo intercultural entre actores con diferentes epistemologías y ontologías sobre los desastres.
\end{abstract}

Palabras claves: Desastres naturales, territorialidad, lafkenche, interculturalidad.

\begin{abstract}
Natural disasters are very common in our country. Earthquakes, tsunamis, volcanic eruptions and floods are phenomena that happens quite frequently, often altering the way local groups interact with nature. This because they are able to modify cultural, social and economic practices reorienting the future of a specific territory, as happened in Saavedra with the $9.5^{\circ}$ earthquake and subsequent tsunami of 1960. From a political ecology of disasters; and through focus groups, semi-structured interviews and participatory mappings; this article analyses the way local authorities with competence in disasters have recognized the relevance of the lafkenche's territoriality, generating an intercultural dialogue between actors that have different epistemologies and ontologies about disasters.
\end{abstract}

Keywords: Natural disasters, territoriality, lafkenche, interculturality.

Artículo recibido el 30 de marzo de 2017, aceptado el 23 de abril de 2018 y corregido el 15 de junio de 2018

2 Centro Nacional de Investigación para la Gestión Integrada de Desastres Naturales (CIGIDEN), CONICYT FONDAP/ Centro de Estudios Interculturales e Indigenas (CIIR), CONICYT FONDAP (Chile).E-mail: fmolinac@gmail.com

3 Observatorio Regional, Universidad Católica de Temuco (Chile). E-mail: dago.constanzo@gmail.com

4. Observatorio Regional, Universidad Católica de Temuco (Chile). E-mail: ccinostroza91@gmail.com 
Chile es un país protagonista en lo que respecta a desastres naturales con efectos catastróficos, los cuales han tenido impacto a nivel nacional e internacional. En los últimos 440 años, en el territorio chileno se han registrado más de 27 terremotos de carácter destructivo (Lagos, 2000; Cisternas et al., 2005; Lagos \& Cisternas, 2008). Asimismo, si consideramos los últimos 60 años, es posible mencionar los mega-terremotos y tsunamis de Valdivia (1960) y Constitución (2010); y los terremotos de la Zona Central (1985), de Arica e Iquique (2014); el tsunami de Aysén (2007); y las erupciones volcánicas de 2008 (Chaitén) y 2011 (Cordón Caulle-Pullehue), los cuales han generado impactos significativos (Mardones \& Vidal, 2001).

Esta situación, adquiere especial relevancia cuando se considera que en Chile existe una gran cantidad de asentamientos humanos (muchos de ellos capitales regionales) y de actividades productivas localizadas en áreas que poseen una alta peligrosidad (Greenhough et al., 2005; Srinivas \& Nakagawab, 2008; Uçklay et al., 2008), lo que promueve la necesidad de generar conocimiento y aprendizaje respecto de estos fenómenos. En esta línea, el presente artículo analiza desde la ecología política y la interculturalidad, el dialogo que se genera entre el pueblo lafkenche y las autoridades públicas en torno a los desastres naturales en la comuna de Saavedra, tomando como contexto las implicancias socioambientales que trajo consigo el terremoto $9,5^{\circ}$ richter y posterior tsunami ocurridos el año 1960.

El documento se divide en cuatro partes. La primera, da cuenta de la aproximación conceptual, donde se analiza desde la ecología política la forma como los diferentes grupos humanos construyen socialmente la naturaleza, el entendimiento de la territorialidad y de los desastres naturales como una manifestación de dicha construcción, además del diálogo intercultural que se da entre diferentes actores en torno a estos fenómenos. La segunda sección, describe en términos generales el método utilizado, haciendo especial hincapié en las razones bajo las cuales Saavedra fue elegido como estudio de caso, las técnicas utilizadas, y el análisis asociado. La tercera sección, en tanto, realiza una reflexión en torno a los principales resultados del artículo. Por último, la cuarta sección del artículo contempla una conclusión que sintetiza los principales argumentos y hallazgos.

\section{La naturaleza como una construcción social}

La denominada "crisis ambiental", ha sido un foco de interés creciente en las ciencias sociales desde los años 1980s, siendo especialmente relevante la relación dialéctica que se da entre naturaleza y sociedad (Blaikie \& Brookfield, 1987; Bryant, 1992). En esta línea, la ecología política ha comenzado a estudiar dicha dialéctica, poniendo énfasis en el rol que tiene la economía política como fuerza de inestabilidad (Walker, 2005). En específico, promueve una visión que rechaza una cultura ecológica apolítica orientada mayormente al medio ambiente (Bryant \& Bailey, 1997; Walker, 2005) y a la adaptación cultural (Peet et al., 2011), asumiendo que la naturaleza es socialmente construida y producida (Bakker \& Bridge 2006), promoviendo así, lo que ha sido denominado "naturaleza social" (Castree, 2001).

De esta forma, la ecología política ha sido capaz de deconstruir esta relación, asumiendo que los discursos y prácticas asociadas corresponden a un proceso de interacción entre instituciones sociales y una heterogeneidad biofísica (Bakker \& Bridge, 2006). Así, ha refutado la idea de que la 
naturaleza se sitúa fuera de las dinámicas sociales, complejizándola y asumiéndola como un conjunto de conocimientos y discursos (Warf, 2004). De la misma forma como ocurre en la ecología cultural y en la antropología ecológica (Biersack, 2006), ha posicionado a la cultura como un elemento central de análisis, realzando la importancia que tienen las representaciones contextuales que los diferentes grupos poseen respecto de ésta (Escobar, 2008).

Así, el considerar la cultura como parte del proceso dialéctico entre naturaleza y sociedad, resulta clave, ya que, permite analizar los patrones culturales que los distintos grupos (en sus respectivas escalas) establecen como prioritarios al momento de relacionarse entre sí y con iniciativas globalizadoras (Swyngedouw, 2004). Asimismo, permite analizar si las iniciativas de desarroIlo que surgen de parte de las agencias del Estado, son culturalmente atingentes al contexto local o buscan apropiarse de éste (Andolina et al., 2009). En términos simples, permite asumir dicha tensión y reflexionar en torno a la capacidad de agencia que tienen los grupos locales para hacer prevalecer estrategias de desarrollo comunitarias y así, acceder a determinadas oportunidades políticas en torno a su relación con la naturaleza (Escobar, 2001; Urkidi, 2010).

Este proceso entre naturaleza y sociedad, no se reduce únicamente a una cuestión epistemológica. Al contrario, desde su tradición posestructuralista, la ecología política ha deconstruido conocimientos valiéndose del método científico (que, también, es su foco de crítica), internándose en debates transformativos y generando cuestionamientos acerca de lo real, surgiendo así una ontología política (Escobar, 2010). Así, ha logrado reconocer que las relaciones dentro del campo de poder que generan una ontología determinada (y por ende una construcción social de la naturaleza), al igual que los conflictos que la subyacen, se encuentran en constante tensión (Blaser, 2009). Por medio de una crítica a la postura multiculturalista de la ontología moderna, al reducir a otras ontologías a la categoría de perspectivas culturales del mundo real (Blaser, 2009), ha propuesto una comprensión del mundo relacional que vincula humanos con no humanos (Escobar, 2012; Escobar, 2015), rompiendo así con la dualidad entre sociedad y naturaleza.

En este contexto, es que se consideran las estrategias de desarrollo comunitarias que posee el pueblo lafkenche frente a los desastres naturales. Desde una ontología relacional que tensiona el statu quo, este pueblo ha posicionado una concepción de mundo, donde los cuerpos de agua son un elemento vital, en tanto son considerados espacios constitutivos de su cultura, religiosidad, sociabilidad y orden social (Rodríguez, 2015). Así, ha sido capaz de posicionar, de hacer dialogar sus sistemas de escala local con sistemas globales "en un contexto [comúnmente] marcado por discursos universalistas e inmovilizantes" (Palomina-Schalscha, 2015: 214).

En concreto, y al analizar las dinámicas que poseen los pueblos indígenas en lo que respecta a desastres naturales, es posible observar que el proyecto neoliberal, como manifestación de dinámicas globalizantes en el entendimiento formal sobre dichos fenómenos, "no es un proyecto inamovible y totalizante" (Palomina-Schalscha, 2015: 229). Al permitir resistencias por medio de prácticas transformativas de reapropiación y negociación, se tensiona no sólo a las estructuras de poder, sino que, también, la toma de decisiones respecto a estos fenómenos. De esta manera, se observa un entrelazamiento de posiciones, a pesar de que el neoliberalismo implica una relación con la naturaleza, que comprende una racionalidad económica que promueve un método de producción y consumo (Bryant \& Goodman, 2003; Leff, 1995), y que ha traído como consecuencia, una ruptura en las antiguas prácticas de subsistencia, que se encuentran basadas en una relación 
de mayor armonía con los recursos naturales (Leff, 2004). No obstante, lo anterior, y para el caso mapuche, no se idealizará el carácter colectivo de la propiedad de la tierra de la sociedad mapuche, ni tampoco en una sacralización de la tierra, asumiendo que ésta, también, se constituye en un sustento económico (Di Giminiani, 2012).

\section{Territorialidad y desastres naturales}

Entendiendo la naturaleza como un proceso de construcción social, es posible señalar que cada sociedad posee una relación particular con ésta, atribuyendo diferentes valores y sistemas de significación (Gonçalves, 2001; Gonçalves \& Leff, 2012) arraigados en su memoria histórica con el territorio, la cual, trae consigo una materialidad, espacialidad y temporalidad determinada (Aliste, 2001, 2008; Bozzano, 2000; Leff, 1994). Por lo mismo, la territorialidad entendida como la conceptualización y significación del espacio (Gonçalves, 2001), adquiere relevancia al entender las diferencias que poseen los grupos indígenas con otros actores no indígenas, tales como las autoridades públicas, en lo que respecta a los ciclos de la naturaleza. Esto, al considerar que la territorialidad incorpora percepciones y distinciones que se dan en dinámicas socionaturales, que incluyen no sólo contenidos materiales, sino, también, simbólicos (Aliste, 2008; Bozzano, 2000), los cuales están embebidos de distinciones tanto epistemológicas como ontológicas.

De esta forma, y para efectos de este artículo, la territorialidad se vinculará con los desastres naturales, al ser entendidos no sólo como eventos naturales, sino que, también, como un producto social, político y económico (Wisner et al., 2004), que responde a complejas relaciones entre el ambiente físico (tanto el natural como el construido) y la sociedad (Birkmann et al., 2013). Si bien, existen diversas aproximaciones al fenómeno de los desastres naturales, donde destacan enfoques de riesgo, estudios sobre vulnerabilidad (Bankoff et al., 2004; Romero Toledo \& Romero Aravena, 2015), y aproximaciones desde la adaptación y resiliencia (Birkmann et al., 2013), el presente artículo busca contribuir a este debate analizando la forma como dichos desastres refuerzan los elementos constitutivos de esta territorialidad, al ser un estímulo que exacerba la relación entre conocimiento y práctica que tienen los grupos humanos indígenas sobre la naturaleza, y que irremediablemente se va traduciendo en conductas consideradas propias (Montemayor, 2000).

En este contexto, los desastres naturales adquieren relevancia, en tanto, demuestran empíricamente la manera como el poseer una determinada territorialidad permite generar procesos de reorganización y aprendizaje (Pelling \& Dill, 2010), adaptándose a los cambios de la naturaleza, al contar con habilidades específicas para moderar sus efectos y enfrentar así, sus perturbaciones (Brooks, Adger \& Kelly, 2005). Asimismo, permite dar cuenta de la forma en que los grupos humanos propensos a enfrentar fenómenos como los desastres naturales han lidiado con la carga política que los Estados les han otorgado a conceptos como la seguridad, la gestión del riesgo y la resiliencia, donde los desafíos de adaptación a estos desastres (en un contexto de cambio climático global) se han constituido en un terreno fértil para reglas neoliberales de gobernanza (Watts, 2015). Por lo mismo, el énfasis de este estudio estará en el entrelazamiento que tiene el pueblo lafkenche con su medio natural, asumiendo su carácter relacional y superando la díada interno-externo (Hallowell, 1955). 
En concreto, la significación que este grupo hace sobre los desastres naturales será vital, ya que permite analizar las fuerzas de la naturaleza como un problema intelectual, no respondiendo al medio ambiente inmediato, sino que a uno de segundo orden que se encuentra culturalizado (Foster \& Holleman, 2012). De esta manera, no se hará hincapié en las significaciones sobre una naturaleza de primer orden (aquella que aparenta ser prístina e intocada por el hombre), sino que se pondrá el foco en una naturaleza de segundo orden, que considere las implicancias que ha traído consigo su transformación en commodities, donde la naturaleza ha sido transformada en bienes comerciales y recursos económicos (Smith, 2008).

\section{Dinámicas interculturales ¿Hacia un diálogo de saberes?}

En este contexto, se observa una gran complejidad en torno a los desastres naturales, la cual está fuertemente mediada por un modelo neoliberal homogenizante y un Estado capaz de generar "contextos institucionales clave" (Whitehead, 2008: 415), que benefician a unos a expensas de otros, y que no consideran procesos de desarrollo local y regional (Swyngedouw, 2004). En lo que respecta al contexto mapuche, el Estado ha tenido un tipo de relación que se apoya en la pobreza material del indígena y no en lo cultural, utilizando "los conceptos multicultural e intercultural para referirse a las relaciones establecidas en el proceso de democratización del país" (Quilaqueo, 2009: 16). Por lo mismo, la interculturalidad será entendida como un proceso dinámico de carácter intersectorial, que no puede ser separado, aislado y yuxtapuesto, y que debe contextualizarse en un proceso histórico determinado (Quilaqueo, 2009).

Cabe señalar, que la cuestión intercultural genera conflictividad. Si bien, para algunos las dinámicas interculturales promueven una relación equitativa entre conocimientos y prácticas, generándose así, tanto una tolerancia de la diferencia, como procesos de intercambio que han permitido construir nuevos espacios de encuentro entre saberes, sentidos y prácticas distintas (Cantle, 2012); para otros la interculturalidad es considerada como un espacio que altera culturas a las cuales no se desea renunciar (Demorgon, 2000), y que segrega, en tanto, se aplica de manera efectiva sólo a los grupos subalternos y no a los que son hegemónicos (Pérez-Aguilera \& Figueroa-Helland, 2011). Esto adquiere relevancia, al darse en un contexto donde los resabios del colonialismo en América Latina han traído consigo una constitución colonial de saberes, lenguajes y de la memoria/imaginario (Quijano, 2000), anclado en un escenario donde la modernidad surge como una cosmovisión imperante (Lander \& Castro-Gómez, 2000). Cosmovisión que ha estado basada, no sólo en una visión de la historia fuertemente asociada a la idea de progreso y a la naturalización de una sociedad liberal capitalista, sino que además, en una donde los saberes que ella misma produce -la ciencia- se han posicionado sobre cualquier otro saber (Lander \& Castro-Gómez, 2000).

Para los efectos de este artículo, la interculturalidad será entendida como un espacio de diálogo que se da entre la cultura dominante (Estado a través de sus autoridades públicas) con la cultura dominada (pueblo lafkenche), con potencial de transformar estructuras e instituciones existentes, promoviendo así, un cambio político y epistémico en torno a los desastres naturales, anclando otros modos de saber, de ser y de poder (Walsh, 2005). Se observa así, una diversidad epistémica capaz de dar cuenta de la existencia de una pluralidad de lugares de enunciación (pasados y presentes) con una relación crítica o de resistencia con dicha modernidad, surgiendo una 
"epistemología de fronteras" (Mignolo, 2007). Esto, asumiendo que la relación entre estos actores no surge como un acoplamiento de dos categorías binarias, sino que, responde a la interacción de múltiples dominios y tipos de conocimiento que poseen diferentes lógicas y epistemologías, que se manifiestan dependiendo de los intereses que los distintos actores persiguen (Agrawal, 1995). Si bien, dicha relación se sustenta en un diálogo asimétrico, supone la reescritura de las narrativas modernas desde un lugar que revaloriza a las culturas y pueblos dominados, así como sus hitos de resistencia (Alimonda, 2011).

Bajo esta lógica, la relación que se ha dado entre el pueblo lafkenche y las autoridades públicas en torno a los desastres naturales, se analizará como una respuesta a las dinámicas multiculturales que subsisten con relativa frecuencia en la Región de la Araucanía (Bolados García, 2012), las cuales perpetúan un modelo de desarrollo que fomenta procesos de desigualdad social, manteniendo estructuras e instituciones que privilegian a unos en desmedro de otros (Walsh, 2005). De esta forma, se releva que la relación que se da entre el pueblo lafkenche y las autoridades públicas no responden necesariamente a relaciones de confrontación y negociación de parte de los pueblos indígenas con el Estado, en el marco de reformas sociales y culturales de carácter neoliberal (Hale, 2002), sino que se constituyen en dinámicas interculturales que facilitan la construcción de modos y condiciones de pensar diferentes, anclados en dinámicas de construcción permanente (Walsh, 2005), que han contribuido a un cambio en la gestión del riesgo en la comuna de Saavedra.

\section{Saavedra y el pueblo lafkenche}

Saavedra es una comuna costera y fluvial ubicada en la Provincia de Cautín, el litoral de la Región de la Araucanía. Tal como se observa en la Figura № 1 , su capital es el centro urbano de Puerto Saavedra y se encuentra inserta en dos cuencas hidrográficas: Ios ríos Budi e Imperial, y posee un borde costero con incipientes llanuras litorales. Esta comuna pertenece tradicionalmente al territorio lafkenche (Lafken: mar; che: gente), el cual poseía como límites el río Imperial por el norte y el río Toltén por el sur (Ruiz \& Figueroa, 2006), y utilizaba la costa como asentamiento por medio de una forma de vida fuertemente vinculada al mar y los recursos marinos (Montalba, 2005).

Esta comuna, se caracteriza por poseer un gran porcentaje de población mapuche. De acuerdo a las cifras de la CASEN 2013, el 84\% de la población son mapuches, de los cuales la mayoría de ella se identifica con el pueblo costero lafkenche, visto como subgrupo (Ministerio de Desarollo Social, 2013). Este pueblo difiere en gran parte de la economía de otros subgrupos del pueblo mapuche y campesina, al estar orientada principalmente a la recolección marina y en menor medida, a la producción agrícola y forestal. Para ellos, el borde costero les otorga una territorialidad basada en el Az Mapu (costumbres de nuestra tierra), donde más allá de la búsqueda del crecimiento económico o la rentabilidad extrema, el propósito recae en una interacción de reciprocidad económica, cultural y social (Chihuailaf, 1999).

En este contexto, es importante señalar que la idea de una cultura rígida y de límites claros pareciera no representar a la cultura mapuche, sin embargo, existen algunos elementos representativos, de los cuales dos son constitutivos de su cultura e identidad: su vinculación con la tierra y su lengua común (Bazán, 2011). Así, la transmisión oral de saberes y conocimientos a las nuevas 
Figura $\mathrm{N}^{\circ} 1$

Área de Estudio, comuna de Saavedra

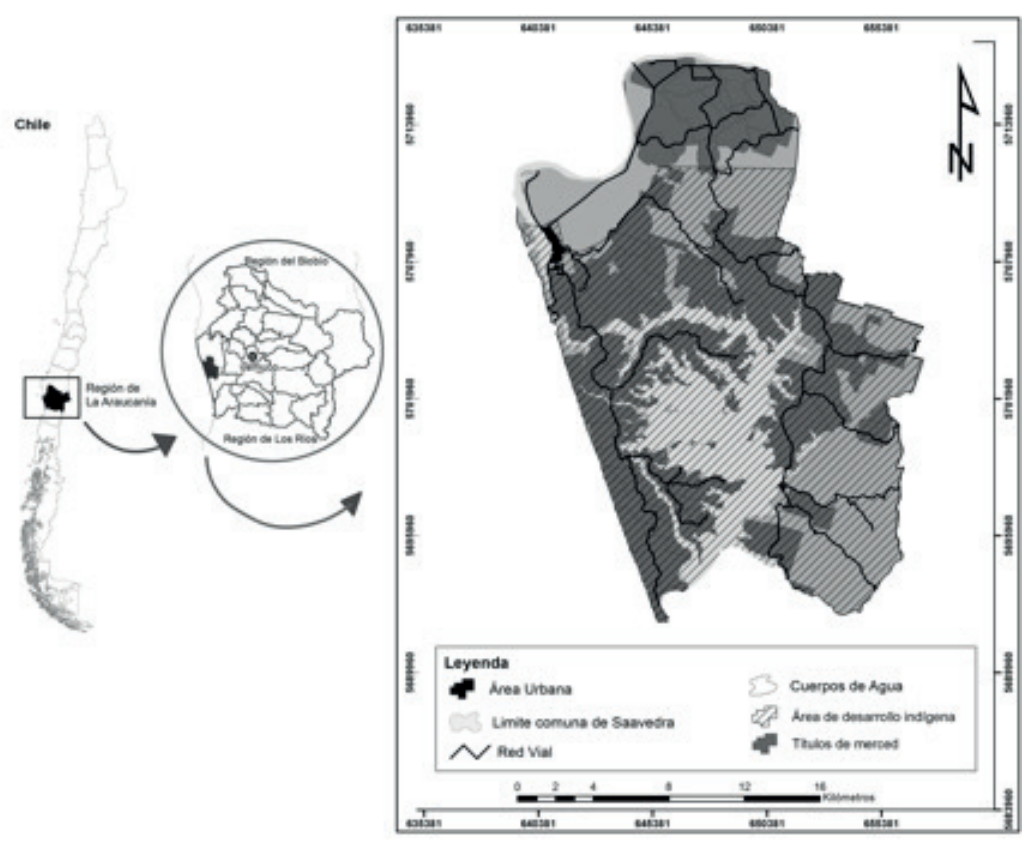

Fuente: Elaboración propia.

generaciones, en el contexto de la familia y la comunidad cobra principal relevancia (Quintriqueo \& Maheux, 2004), dado que permiten la interacción de las personas con el entorno social, natural y espiritual de acuerdo a las pautas culturales mapuches establecidas (Quilaqueo et al., 2005). El mapuzungun (lengua mapuche) es vital en la formación de una lógica de cocimiento que permite dar significado a los elementos culturales mapuche y no mapuche, a través de una determinada apropiación cultural (Quilaqueo et al., 2005; Quilaqueo, 2007), constituyendo saberes que forman parte de un sistema simbólico.

Así, los saberes que se emplean se sustentan en la memoria social (Le Goff, 1991; MontespereIli, 2004), que, entre los kimches, definido en mapunzugun como sabio, docto y erudito (Augusta, 1903), se manifiesta en segmentos específicos de enseñanza. Así, se logran actitudes para construir y mantener el saber de la comunidad, tal como sucede con los desastres naturales, donde el conocimiento posee características comunes y accesibles a sus miembros. En otras palabras, la memoria no es sólo un acervo de conocimiento interior a cada individuo, sino, que se proyecta al exterior. Ésta se vuelve colectiva e intersubjetiva al ser compartida, ya que va más allá de su objetivación (Berger \& Luckman, 2002; Montesperelli, 2004).

Esta fuerte presencia indígena y acervo cultural es administrativamente reconocida por parte del Estado. Tal como se observa en la Figura $\mathrm{N}^{\circ} 2$, la comuna de Saavedra pertenece al Área de Desarrollo Indígena (ADI) Lago Budi, la cual fue establecida el 23 de abril de 1997 a través el decreto $\mathrm{N}^{\circ} 71$, y que corresponde a "espacios territoriales en que los organismos del Estado focalizarán su acción en beneficio del desarrollo armónico de los indígenas y sus comunidades" (Ley 19.253, 
Párrafo 2, artículo $26^{\circ}$ ). En esta línea, se caracterizan por poseer un conocimiento y uso ancestral del espacio costero marino, el cual, se encuentra resguardado en la Ley 20.249 "Espacio costero marino de los pueblos Originarios", cuyo objetivo es mantener las tradiciones y uso ancestral de dichos recursos por parte de las comunidades vinculadas al borde costero (Ley 20.249, 2008).

Figura $\mathrm{N}^{\circ} 2$

Mapa comunitario de riesgos socionaturales, comuna de Saavedra

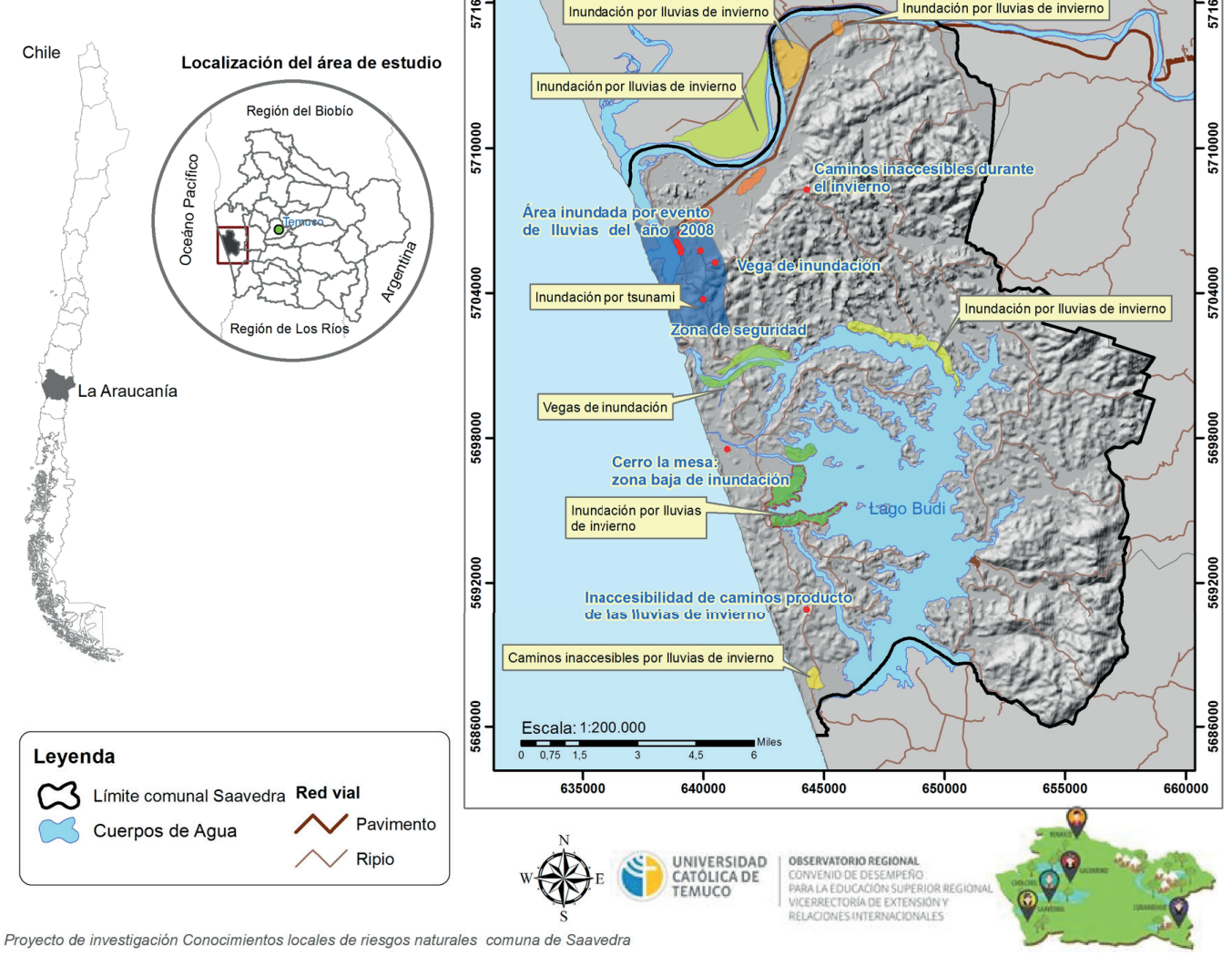

Fuente: Elaboración propia.

Este particular interés del Estado, también, se debe a que la comuna de Saavedra se emplaza en terrenos afectos históricamente a desastres naturales, siendo un hecho emblemático el terremoto y posterior tsunami de 1960. En esta línea, mientras a escala de impacto macro se identifican tsunamis y efectos de terremoto, a escala local destacan las inundaciones y anegamientos producto del desborde del canal 21 de mayo, además de aquellos generados por la conjunción del crecimiento de la laguna Imperial y de las napas subterráneas cercanas a la superficie (ver Figura $\mathrm{N}^{\circ} 2$ ). Pese a lo anterior, es posible observar una falta de consolidación y planificación del crecimiento urbano en las zonas seguras, lo que se traduce en que existe una reconstrucción incompleta no sólo de los impactos que tuvo el terremoto de febrero de 2010, sino que, también, de aquellos asociados al tsunami de mayo de 1960. 
Si bien, este escenario se constituye en un terreno fértil para dinámicas de gobernanza poco inclusivas, el conocimiento local que posee el pueblo lafkenche ha sido gradualmente incorporado en la gestión del riesgo que se ha definido para la comuna de Saavedra. Para efectos de este artículo, la gestión del riesgo se entenderá como un proceso dinámico que busca prevenir y corregir aspectos generadores de riesgo (prospectivos y de prevención), prestar atención a los efectos de un evento desastroso (asistencia) y realizar las acciones encaminadas a la reconstrucción (Hernández \& Vargas, 2015). En este caso, el riesgo, se constituye en una apreciación subjetiva del individuo frente a una situación determinada, influenciada por la visión que sostenga sobre la naturaleza y la experiencia que tiene como sujeto social, antes que un producto de análisis racional (Losada, 2010).

Al entender la visión del riesgo como una instancia de gestión, adquiere relevancia la incorporación de diferentes actores sociales que pueden fortalecer los conocimientos institucionalizados. Para efectos de este artículo, la incorporación de los saberes locales y su intercambio con los conocimientos técnicos ya establecidos (Losada, 2010) surge como una posibilidad para generar una visión respecto del territorio, que permita tomar decisiones geográfica y culturalmente atingentes sobre esta materia.

\section{Método}

La aproximación posee un carácter cualitativo orientado a comprender la perspectiva de los participantes sobre los fenómenos que los rodean, además de profundizar en las experiencias, perspectivas, opiniones y significados que éstos poseen sobre su realidad (Hernández-Sampieri et al., 2010). Así, lo que se busca es conocer el pensamiento local asociado a necesidades, valores e intereses (Piaget, 1976) y los aspectos que son relevantes para una determinada área de estudio (Corbin \& Strauss 1990). En términos concretos, se consideró un método de investigación (Grounded Theory) que permite una teoría que surja desde los datos (Glaser et al., 1967).

En este contexto, se llevaron a cabo 2 grupos focales a representantes lafkenche de la comuna de Saavedra ${ }^{5}, 8$ entrevistas semi-estructuradas a autoridades con competencia en desastres naturales, y se elaboraron 2 mapeos participativos con instituciones públicas y representantes de la sociedad civil. Los grupos focales se realizaron con el propósito de conocer la forma como los lafkenche generan una perspectiva de los desastres naturales, por medio de su interacción en grupo (Hernández Sampieri et al., 2010). Para esto, se hizo hincapié en su interacción (Barbour, 2007), con el objetivo de analizar la aproximación que tienen sobre dichos fenómenos. Las entrevistas semi-estructuradas, en tanto, se efectuaron con el propósito de incorporar preguntas adicionales que permitiesen precisar conceptos y obtener información sobre áreas de mayor sensibilidad (Hernández Sampieri et al., 2010). Para esto, se seleccionó a representantes de instituciones públicas con alcance comunal, que tienen competencias sobre desastres naturales. 
Asimismo, el mapeo participativo ${ }^{6}$ fue utilizado para obtener información basada en el conocimiento local, otorgándole así un especial valor al conocimiento intrínseco que poseen los actores. En este marco, se desarrollaron 2 mesas de trabajo, en las cuales, participaron representantes tanto de la sociedad civil como de instituciones públicas, con el propósito de levantar información complementaria a las entrevistas y los grupos de discusión, basada en el contexto (Brown \& Fagerholm, 2014). Esto, considerando que esta técnica permite comprender el contexto de la vida cotidiana de las personas (Kyttä, 2011), constituyéndose en un proceso creativo de producción socioespacial, basado en las realidades endógena. En otras palabras, que es construido a través de múltiples formas de representación, traduciendo y haciendo visible el entendimiento y la comprensión local (Sletto et al., 2013).

Por último, para el análisis de los datos se trabajó en tres categorías centrales que se vinculan con los conceptos analizados en este artículo: relación naturaleza y sociedad, territorialidad y desastres naturales, y dinámicas interculturales. Esto, con el objeto de estructurar los datos ( $\mathrm{Pa}$ tton, 2002) y de describir las experiencias de las personas que participaron de las instancias de levantamiento de información, intentando identificar la óptica desde donde se analizan (Creswell, 2009). En concreto, este enfoque de trabajo se ampara en la idea de que tradiciones, roles, valores y normas al ser internalizadas van generando regularidades que explican la conducta de ciertos individuos y grupos. Asimismo, orienta su análisis hacia dichas regularidades, al asumir que los lafkenche comparten una estructura lógica o de razonamiento (Martínez, 2004).

\section{Diferentes territorialidades para un mismo espacio}

Tal como fue mencionado en la sección anterior, el pueblo lafkenche posee una relación particular con la naturaleza, especialmente con los cuerpos de agua. En este sentido, posee una materialidad, espacialidad y temporalidad que está determinada por las dinámicas socionaturales que ancestralmente han tenido sobre el territorio, destacando especialmente el Lago Budi, el cual posee un importante valor cultural, ambiental y turístico (Escalona-Ulloa et al., 2012). Asimismo, la gran cantidad de sitios de significación cultural (ver Figura $\mathrm{N}^{\circ} 3$ ) denota un conjunto de representaciones y de apropiaciones simbólicas y materiales (Aliste, 2008; Bozzano, 2000), que dan cuenta de la importancia que este territorio tiene para este pueblo. En contraste, y si analizamos la vinculación con el territorio que tienen las instituciones públicas con competencia en materia de desastres, se puede observar que no existe ese tipo de apropiación y que las representaciones que hacen sobre éste, demuestran un desarraigo mayor: "Por ejemplo yo soy de Temuco, y llevo dos años y medio acá y no sé si voy a estar dos años y medio más" (Autoridad comunal 1, 2015).

Para la realización de este mapeo se utilizó un mapa en papel de $120 \mathrm{~cm} \times 100 \mathrm{~cm}$, en los que se presentaron los límites administrativos de la comuna, los cursos y cuerpos de agua, la red vial, y la toponimia (nombre de lugares). Además, se proyectó una imagen satelital de fondo con la finalidad de familiarizar a los actores con el instrumento. Durante el relato se solicitó a los participantes identificar la localización del territorio y los riesgos a los cuales está expuesto la comuna, los cuales fueron demarcados por medio de polígonos o puntos. Una vez ejecutado el proceso, se escanearon las cartografías y apoyado en el sistema de información geográfico Arcgis 10.1, se georreferenciaron y digitalizaron los mapas antes escaneados, de modo de obtener una versión exacta de la zonificación realizada por los participantes del taller. 
Figura $\mathrm{N}^{\circ} 3$

Sitios de significación cultural

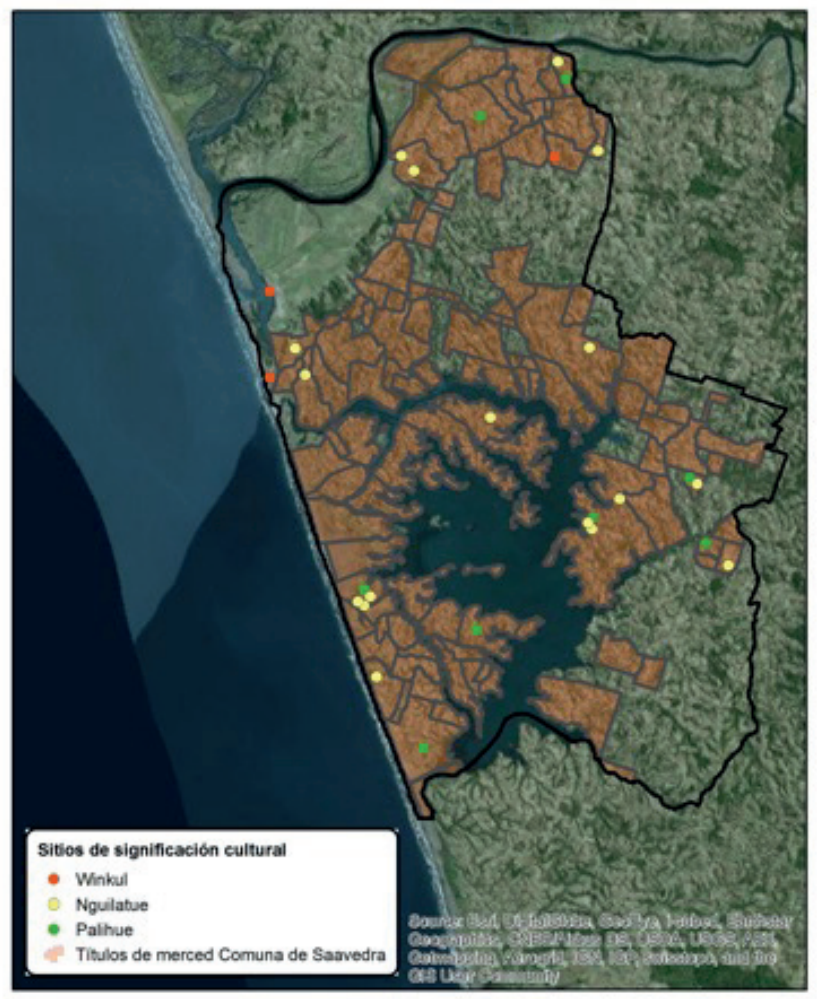

Fuente: Elaboración propia.

En esta línea, la aproximación que tienen las autoridades públicas está más orientada a las consecuencias económicas que trajo consigo el terremoto y posterior tsunami de 1960, lo que para ellos ha sido el principal causante de un estancamiento en otras áreas: "Hacia antes del año 60 (...) teníamos a bastantes extranjeros que te movían la economía de Saavedra, y después de los 60, esta gente se fue, y se generó una depreciación de las vías económicas de Saavedra" (Autoridad comunal 3, 2015). De esta manera, su rol está orientado a buscar los mecanismos de seguridad que permitan a la comuna surgir nuevamente, siendo el turismo la principal fortaleza: "[Desde hace] 10 años Saavedra está levantándose gracias al turismo" (Autoridad comunal 3, 2015).

En este marco, al analizar la territorialidad de los distintos actores y su vinculación con los desastres naturales, es posible observar que las instituciones públicas de Saavedra poseen patrones culturales diferentes a los que posee el pueblo lafkenche, al tener una pertinencia territorial distinta. En concreto, ellos asumen su condición de afuerinos: "[Entre el] 50\% y $60 \%$ de los directivos somos de afuera (...), hay harta gente que no es permanente (...), los marinos son tipos que están asignados, por 3-4 años, y después llegan y se van y así digamos la institución estatal, es un personal flotante acá en Puerto Saavedra" (Autoridad comunal 1, 2015). Al aceptar esta condición, la conceptualización y significación que los actores tienen de la comuna de Saavedra (como espacio de análisis), y de la potencial presencia de desastres naturales asociados, se realiza desde 
perspectivas diferentes. Por una parte, las autoridades públicas poseen una relación con el territorio de carácter estrictamente funcional, abocada al número de personas que se requieren para garantizar la seguridad: "En Puerto Saavedra, no hay tantos profesionales y técnicos, entonces tenemos que venir de afuera a cumplir esa función" (Autoridad comunal 1, 2015). No obstante, lo anterior, son enfáticos en argumentar que ellos poseen un mayor vínculo en la ciudad de Temuco (capital regional), lugar donde se encuentran sus familiares y donde realizan sus actividades cotidianas. En concreto, manifiestan que, ante la presencia de un desastre natural, ellos privilegiarían centrar sus energías en esa ciudad: "Si el evento ocurre un día en la noche, un día sábado, yo voy a salvar a mi familia en Temuco" (Autoridad comunal 1, 2015).

Por otra parte, los representantes del pueblo lafkenche poseen una vinculación mayor con este tipo de fenómenos, que está amparado en la transmisión de conocimiento, que de generación en generación, se ha hecho a nivel local'. Las marcas que presentan la actual configuración del paisaje producto del terremoto y tsunami de 1960, o la fuerza de Kai Kai Filu (serpiente que representa la negatividad de la cosmovisión mapuche), es un recuerdo cotidiano para ese pueblo, tanto, que modificó hasta el día de hoy sus relaciones socioculturales. Por lo mismo, se manifiesta una fuerte admiración hacia los adultos mayores, al haber sido capaces de sobrellevar las consecuencias del tsunami de 1960: "Recordando el pasado y el año 60 (...), mi mamá tiene una historia muy bonita (...), ella dice sufrieron (...), pero es valiente, [ella] conoce lo que es [el afrontar un desastre] y eso es bonito para uno cuando conversa con una persona adulta" (Representante lafkenche 2, 2015). De esta manera, se manifiesta una cercanía con la historia reciente que posee la comuna, lo que los vincula simbólicamente con el territorio.

\section{Ocupación del territorio y búsqueda de sinergias}

La forma como se distribuye el pueblo lafkenche en el territorio, es una manifestación concreta del conocimiento que poseen sobre éste. En esta línea, el emplazamiento de los asentamientos humanos cercanos a los sitios de seguridad, donde destaca el cerro La Mesa, sitio en el cual se agrupó la comunidad ante el tsunami de 1960, es una clara demostración de cómo íconos del acervo cultural lafkenche, han sido trasmitidos de generación en generación: "Las personas mayores lo transmiten, porque ellos realmente lo vivieron, entonces ahí se juntaron, en una parte donde se hacen ceremonias culturales" (Representante lafkenche 3, 2015). Esto, es ampliamente reconocido por parte de las instituciones públicas, las cuales asumen que los lafkenche poseen una apreciación del territorio diferente. En esta materia, además de valorar positivamente la educación formal que se entrega en las escuelas respecto de los desastres naturales, destacan el proceso formativo que existe en las familias sobre la temática, lo que ha generado procesos de reorganización y aprendizaje (Pelling \& Dill, 2010): “Hablemos (...) del tsunami, que es el más riesgoso, se enseña en las escuelas, y hay otro tema que se ha traspasado de generación en generación, es decir, hay un trabajo de las mismas familias" (Autoridad comunal 2, 2015).

Cabe señalar que este conocimiento no se reduce únicamente a los desastres naturales. En este sentido, la relación de individuos con el medio natural le ha permitido comenzar a reconocer los efectos del cambio climático en la zona. Sobre esto cabe señalar que, al estar la base productiva de la comuna orientada a la actividad agrícola de papas y trigo, se ha acrecentado un déficit hídrico: "Los pequeños agricultores te dicen que hoy da los cultivos no están rindiendo lo que tienen que rendir (...) es la condición de déficit hídrico y de cambio climático está generando un problema" (Autoridad comunal 5, 2015). 
Asimismo, reconocen que el conocimiento del pueblo lafkenche tiene sobre la zona les ha permitido compensar la fuerte vulnerabilidad que existe en la comuna, donde el grueso de la población y los servicios se encuentran en zonas de riesgo: "Si hay una catástrofe como pasó para el año 60, donde desapareció Puerto Saavedra, [podría pasar lo mismo hoy en día ya que] están viviendo en la misma zona" (Autoridad comunal 3, 2015). En otras palabras, les ha entregado nuevas herramientas para afrontar la falta de estándares que ha tenido el diseño y emplazamiento de los sistemas de seguridad con que cuenta actualmente la comuna: "Lamentablemente acá todos los sistemas y servicios se encuentran en zonas de riesgo, por decir lo menos, hasta la sirena se llevaría en caso de un tsunami en la comuna" (Autoridad comunal 7, 2015).

No obstante lo anterior, aún se mantiene una percepción de inseguridad en algunos grupos de la comunidad, debido a los episodios de inundación de los centros urbanos por la crecida de ríos y canales, y al derrumbe de infraestructura producto de los terremotos. Esto, ha llevado a las autoridades a tomar medidas correctivas con el objeto de hacer validas estas demandas de la población local: "Hemos hecho un gran esfuerzo implementando dos refugios en zonas seguras, en zonas altas, que permiten que la población en caso de emergencia vaya con toda confianza, además, se está año a año trabajando en lo del canal 21 de mayo y se trabaja más consciente de lo que puede pasar" (Autoridad comunal 1, 2015). Igualmente, han fortalecido el equipamiento tecnológico que posee la Armada en la materia, constituyéndose en un actor relevante al igual que la municipalidad: "Acá está la armada y ellos tienen la tecnología necesaria, por que trabajan directo con la ONEMI, y también la municipalidad está comprando siempre cosas para los refugios" (Representante lafkenche 3, 2015).

Este esfuerzo para fortalecer sus sistemas de emergencia se ha anclado en el conocimiento que tiene el pueblo lafkenche sobre las zonas rurales: "Ellos se conocen todo, ve que muchos llegan caminando acá, entonces saben dónde hay un lugar de refugio, donde se inunda todo" (Autoridad comunal 4, 2015). Una manifestación de dicho conocimiento, y que se encuentra vinculado a las actividades agropecuarias de subsistencia que posee el pueblo lafkenche, tiene relación con la tenencia de alimentos, agua y medicamentos en las zonas seguras: "Aquí la gente ha aprendido muy bien, ha aprendido a estar preparado, pero como ciudadanos y campesinos hemos aprendido a tener nuestro bolsito, a cambiar los medicamentos a bolsas con agua, al saco de dormir, a todo eso" (Representante lafkenche 2, 2015).

En síntesis, se observa una sinergia creciente entre el pueblo lafkenche y las instituciones públicas encargadas de afrontar desastres naturales, tal como lo señala un representante de la comunidad: "Ellos nos pasan celulares, nos capacitan y sabemos cómo nos dicen que tenemos que hacer, ahora hasta tenemos una radio en las islas para la gente vive allá" (Representante lafkenche 6 , 2015). Así, se han sustentado las bases para un trabajo conjunto anclado en un intercambio de saberes.

\section{Autoridades y saberes locales: hacia un dialogo intercultural}

En este contexto de intercambio, se observa que las instituciones públicas han sabido reconocer el saber local que el pueblo lafkenche posee frente a los desastres naturales, tal como queda 
de manifiesto en este testimonio sobre los procesos de evacuación realizados en los años 2010 y 2015: "[Para] los dos últimos terremotos, el del 2010 y [el de] ahora, la gente fue oportunamente evacuada, pero si (...) hubiese pasado algo, todos se salvaban (...) porque hay una conciencia de este tipo de fenómenos" (Autoridad comunal 2, 2015). En esta línea, hay tres niveles en los cuales se observan dinámicas interculturales, donde prima una relación equitativa entre los conocimientos y las prácticas (Cantle, 2012), como el que posee el pueblo lafkenche y el conocimiento formal propuesto por las instituciones públicas en sus planes de emergencia. De esta forma, se observa una interacción que proviene de múltiples dominios y tipos de conocimiento (Agrawal, 1995) que encuentran un interés común en la gestión del riesgo: "Por una parte, ellos [pueblo lafkenche] tienen el conocimiento que se ha transmitido, y por otra, en conjunto con nosotros [instituciones públicas], saben qué medidas tomar (Autoridad comunal 8, 2015).

En primer lugar, las instituciones públicas reconocen la "naturalidad" que tiene el pueblo lafkenche frente a este tipo de desastres, dando cuenta explícitamente de la forma como estos fenómenos se constituyen como un producto social, político y económico (Wisner et al., 2004) que adquiere particularidades diferentes dependiendo de cada actor. El caso de los adultos mayores, es destacado especialmente, ya que, al ser testigos del terremoto y posterior tsunami de 1960, dan cuenta de una aceptación frente a las perturbaciones y episodios de estrés asociados a estos desastres, demostrando importantes rasgos de aprendizaje: "Los abuelitos, lo ven como algo que no se puede contener y no se puede prevenir, evitar, ni mitigar, sino que, lo ven como algo que va a suceder y que depende casi de voluntad divina" (Autoridad comunal 3, 2015). Así, la "naturalidad" frente a los desastres naturales les permite una mejor coexistencia con éstos, que se materializa en acciones de gestión del riesgo que son más eficientes que las de los no lafkenche. Un ejemplo concreto, es el emplazamiento de acopio de forraje en zonas seguras. Esto, dialoga con el hecho de que en la actualidad existe mayor tranquilidad respecto de las capacidades que el pueblo lafkenche posee a nivel local: "Yo creo que hemos ido aprendiendo [lo] que tenemos que hacer, (...) después del terremoto del 60 (...) [había] mucha gente que no sabía lo que era un terremoto" (Representante lafkenche 1, 2015).

En segundo lugar, las autoridades también valoran el "liderazgo" que poseen los dirigentes locales al momento de comunicar los alcances que tienen los planes de emergencia oficiales: "Acá en la comuna de Saavedra, los dirigentes cumplen un papel importante y son ellos canales de bajada de información, no sé si los únicos, pero son complementarias digamos a otras acciones que uno puede desarrollar" (Autoridad comunal 6, 2015). Asimismo, reconocen los saberes que poseen en materia de seguridad: "cuando se hacen las consultas de paz ciudadana o cosas así, la comuna tiene clarito, oye que faltan sirenas, falta apoyo, el refugio (...) entonces la comunidad ya sabe lo que tiene y lo que le falta (Autoridad comunal 4, 2015). En concreto, se observa una autocrítica de parte de las autoridades, las cuales asumen que las instituciones formales también tienen el riesgo de equivocarse, reconociendo explícitamente que el rol que tienen los dirigentes es fundamental, ya que, no sólo son conocedores del territorio y de sus particularidades, sino que también, son validados localmente. Por lo mismo, esperan una participación más activa de ellos: "Es una cuestión (...) de todos [estar preparados frente a los desastres naturales], entonces si ellos son capaces de resolver sus problemas, le quitan esta responsabilidad a otras instituciones públicas que pudiesen fallar en algún momento (Autoridad comunal 1, 2015). En esta línea, reconocen que el conocimiento que los adultos mayores poseen sobre el terremoto y posterior tsunami de 1960 ha sido clave para crear conciencia en los más jóvenes, mejorando así la gestión de los ries- 
gos: “En 15 minutos, más del 90\% del pueblo estaba evacuado, el año 2010. Es que acá tenemos la garantía de que los más viejitos vivieron lo del 60" (Autoridad comunal 7, 2015).

Por último, reconocen la fortaleza que posee el pueblo lafkenche al momento de "transmitir" los saberes locales y las medidas de seguridad, aludiendo al hecho de que cuentan con un tejido social fuertemente constituido. En este sentido, la comunicación efectiva de los desastres naturales y el mapuzungun como medio para llevar a cabo dicha comunicación, han generado una preparación exitosa que es explícitamente reconocida por la autoridad: "En cuanto al tejido social, es súper importante para nosotros que en las comunidades hablen su idioma, el mapuzungun, porque así ellos mismos se transmiten el mensaje de mejor forma, [a] que vayamos nosotros a decirles hagan esto, hagan lo otro" (Autoridad comunal 2, 2015).

\section{Conclusión}

Asumir que la naturaleza es construida socialmente, permite relevar la importancia que tienen los patrones culturales bajo los cuales, cada grupo humano realiza dicha construcción. Esto permite reconocer, que los distintos actores tienen territorialidades particulares, las cuales están embebidas de distinciones, tanto epistemológica como ontológicamente (Escobar, 2012, 2015), y responden a los contenidos materiales y simbólicos que le asignan (Gonçalves, 2001). En el caso del pueblo lafkenche, la conceptualización que tienen sobre los desastres naturales, se constituye en una manifestación empírica de una territorialidad que les ha permitido generar determinados procesos de reorganización y aprendizaje (Pelling \& Dill 2010).

Así, este artículo reflexiona en torno al reconocimiento que las autoridades públicas han tenido, respecto del conocimiento local del pueblo lafkenche en materia de desastres naturales. $\mathrm{Si}$ bien, los desafíos de la adaptación en esta materia, han suscitado un interés creciente en un contexto neoliberal global (Watts, 2015), donde la relación del Estado se ha enfocado históricamente en la pobreza material de pueblo mapuche (Quilaqueo Rapimán, 2011), este reconocimiento ha generado una diálogo intercultural que ha permitido la creación de un espacio de encuentro entre saberes, sentidos y prácticas, generando sinergias que han sido capaces de fortalecer los planes de emergencia comunales en materia de desastres naturales, poniendo en valor el conocimiento local.

Esta línea, permite fortalecer y complementar las diversas aproximaciones al fenómeno de los desastres naturales y repensar el rol que tiene la territorialidad, al reflexionar localmente sobre riesgo, vulnerabilidad, adaptación y resiliencia. Esto, considerando que los escenarios de riesgo son fenómenos dinámicos y complejos que exigen el reconocimiento, tanto, de quién interpreta un escenario científicamente, como de quién lo interpreta por medio de los saberes locales (Wilches-Chaux, 1998). Por lo mismo, la valoración de los lafkenche de Saavedra no sólo tiene una relevancia conceptual, sino que, también, ha comenzado a traducirse en acciones, comportamientos prácticos y en proceso de decisiones, permitiendo afrontar adecuadamente determinadas situaciones. Por último, se aprecia un proceso de conocimiento emergente en la comuna, que puede proporcionar ciertos elementos de auto reconocimiento en pos de la construcción de métodos auto-organizativos para afrontar eventos de estas características (Hernández \& Vargas, 2015). Así, el reconocimiento de los saberes locales como información válida, puede nutrir los 
instrumentos de planificación local y la toma de decisiones sobre el territorio, e incidir en escalas territoriales más amplias.

\section{Referencias bibliográficas}

AGRAWAL, A. Dismantling the divide between indigenous and scientific knowledge. Development and change, 1995, Vol. 26, p. 413-439.

ALIMONDA, H. (coordinador). Naturaleza colonizada. Buenos Aires: Ediciones CICCUS, 2011.

ALISTE, E. Lo Ambiental y la Necesidad Histórica en las Sociedades de Riesgo. Revista electrónica MAD, 2001, N5. Disponible en Internet: https://revistas.uchile.cl/index.php/RMAD/article/ view/14822

ALISTE, E. Aspectos sociales y culturales del desarrollo local: desafíos en la planificación y gestión ambientalmente sustentable del territorio. En: FUERTES, A. \& GATICA, L. (editores). De la economía global al desarrollo local. El alcance de la intervención de los agentes de empleo y desarrollo local. Valencia: Ediciones de la Universitat de Valencia, 2008, p. 85-95.

ANDOLINA, R.; LAURIE, N. \& RADCLIFFE, S. Indigenous development in the Andes: culture, power, and transnationalism. Durham and London: Duke University Press, 2009.

AUGUSTA, A. Gramática araucana. Valdivia: Imprenta Central J. Lampert, 1903.

BAKKER, K. \& BRIDGE, G. Material worlds? Resource geographies and the 'matter of nature. Progress in Human Geography, 2006, Vol. 30, p. 5-27.

BANKOFF, G.; FRERKS, G. \& HILHORST, D. (editores). Mapping vulnerability: disasters, development and people. London: Earthscan, 2004.

BARBOUR, R. Doing focus groups. London: Sage, 2007.

BAZÁN, J. ¿Es mapuche el conflicto? Santiago de Chile: Maye Ltda., 2011.

BERGER, P. \& LUCKMANN, T. La Construcción Social de la Realidad. Madrid: Amorrortu Editores, 2001.

BIERSACK, A. Reimagining Political Ecology: Culture/Power/History/Nature. In: BIERSACK, A. \& GREENBERG, J. (editores). Reimagining Political Ecology, United States of America: Duke University Press, 2006, p. 3-40.

BIRKMANN, J.; CARDONA, O.; CARREÑO, M.; BARBAT, A.; PELLING, M.; SCHNEIDERBAUER, S.; KIENBERGER, S.; KEILER, M.; ALEXANDER, D. \& ZEIl, P. Framing vulnerability, risk and societal responses: the MOVE framework. Natural hazards, 2013, Vol. 67, p. 193-211. 
BLAIKIE, P. \& BROOKFIELD, H. (editores). Land degradation and society. London: Methuen, 1987.

BLASER, M. La ontología política de un programa de caza sustentable. WAN e-journal, 2009, Vol. 4, p. 81-107.

BOLADOS GARCÍA, P. Neoliberalismo multicultural en el Chile postdictadura: la política indígena en salud y sus efectos en comunidades mapuches y atacameñas. Chungará, 2012, Vol. 44, p. 135144.

BOZZANO, H. Territorios reales, territorios pensados, territorios posibles: aportes para una teoría territorial del ambiente. Buenos Aires: Espacio editorial, 2000.

BROOKS, N.; ADGER, N. \& KELLY, M. The determinants of vulnerability and adaptive capacity at the national level and the implications for adaptation. Global environmental change, 2005, Vol. 15, p. 151-163.

BROWN, G. \& FAGERHOLM, N. Empirical PPGIS/PGIS mapping of ecosystem services: a review and evaluation. Ecosystem Services, 2015, Vol. 13, p. 119-133.

BRYANT, R. Political ecology: An emerging research agenda in Third World studies. Political Geography, 1992, Vol. 11, p. 12-36.

BRYANT, R. \& BAILEY, S. Third World Political Ecology. London: Routledge, 1997.

BRYANT, R. \& GOODMAN, M. Consuming narratives: the political ecology of 'alternative' consumption. Trans Inst Br Geogr, 2003, Vol. 29, p. 344-366.

CANTLE, T. Interculturalism: The new era of cohesion and diversity. Great Britain: Palgrave MacmiIlan, 2012.

CASTREE, N. Socializing nature: Theory, practice, and politics. In: CASTREE, N. \& BRAUN, B. (editores). Social nature: Theory, practice, and politics. Oxford: Blackwell, 2001, p. 1-21.

CHIHUAILAF, E. Recado confidencial a los chilenos. Santiago de Chile: LOM, 1999.

CISTERNAS, M.; ATWATER, F.; TORREJÓN, F.; SAWAI, Y.; MACHUCA, G.; LAGOS, M.; EIPERT, A.; YOULTON, C.; SALGADO, I.; KAMATAKI, T.; SHISHIKURA, M.; RAJENDRAN, C. P.; MALIK, J.; RIZAL, Y. \& HUSNI, M. Predecessors of the giant 1960 Chile earthquake. Nature, 2005, Vol. 437, p. 404-407.

CORBIN, J. \& STRAUSS, A. Basics of qualitative research: Grounded Theory procedures and techniques. E.E.U.U: Sage Publications, Inc., 1990.

CRESWELL, J. Research design: Quali tat ive, quantitative and mixed approaches. EEUU: Sage, 2009.

DEMORGON, J. Complexité des cultures et de l'interculturel. Paris: Anthropos, 2000. 
DI GIMINIANI, P. Tierras ancestrales, disputas contemporáneas: Pertenencia y demandas territoriales en la sociedad Mapuche rural. Santiago de Chile: Ediciones UC, 2012.

ESCALONA-ULLOA, M.; PEÑA, F. y HIRIARTE, R. Oferta turística y perfil sociocultural de los empresarios turísticos del borde costero de la Araucanía, Chile. Estudios y perspectivas en turismo, 2012, Vol. 21, p. 306-321.

ESCOBAR, A. Culture sits in places: Reflections on globalism and subaltern strategies of localization. Political Geography, 2001, Vol. 20, p. 139-174.

ESCOBAR, A. Territories of Difference. Place, movements, life, redes. Durham and London: Duke University Press, 2008.

ESCOBAR, A. Ecologías políticas postconstructivistas. Revista Sustentabilidades, 2010, Vol. 2. Disponible en Internet:

http://www.sustentabilidades.usach.cl/sites/sustentable/files/paginas/02-05.pdf

ESCOBAR, A. Cultura y diferencia: la ontología política del campo de Cultura y Desarrollo. Wale'keru, 2012, № 2. Disponible en Internet:

https://dugi-doc.udg.edu/bitstream/handle/10256/7724/WALEKERU-Num2-p7-16.pdf?sequence=1\&isAllowed $=y$

ESCOBAR, A. Territorios de diferencia: la ontología política de los "derechos al territorio". Cuadernos de antropología social, 2015, $N^{\circ} 41$, p. 25-38.

FOSTER, J. \& HOLLEMAN, H. Weber and the Environment: Classical Foundations for a Postexemptionalist Sociology1. American Journal of Sociology, 2012, Vol. 117, p. 1625-1673.

GLASER, B; STRAUSS, A. \& STRUTZEL, E. The discovery of grounded theory; strategies for qualitative research. Nursing research, 1968, Vol. 17, $N^{\circ} 4$, p. 364.

GONÇALVES, C. Geo-grafias: movimientos sociales, nuevas territorialidades y sustentabilidad. Mexico: Siglo XXI, 2001.

GONÇALVES, C. \& LEFF, E. Political Ecology in Latin America: the social reappropriation of nature, the reinvention of territories and the construction of an environmental rationality. Desenvolvimento e Meio Ambiente, 2015, Vol. 35, p. 65-88.

GREENHOUGH, B.; JAZEEL, T. \& MASSEY, D. The Indian Ocean tsunami: geographical commentaries One year on. The Geographical Journal, 2005, Vol. 171, N 4, p. 369-386.

HALE, C. Does multiculturalism menace? Governance, cultural rights and the politics of identity in Guatemala. Journal of Latin American Studies, 2002, Vol. 34, p. 485-524.

HALLOWELL, A. Culture and experience, Baltimore: University of Pennsylvania Press, 1955. 
HERNÁNDEZ SAMPIERI, R.; FERNÁNDEZ COLLADO, C. \& BAPTISTA LUCIO, P. Metodología de la investigación. México: Editorial Mc Graw Hill, 2010.

HERNÁNDEZ, P. \& VARGAS, G. Hacia la construcción de conocimiento emergente para la gestión local del riesgo. Cuadernos de Geografía, 2015, Vol. 24, p. 15-34.

KYTTÄ M. SoftGIS methods in planning evaluation. Evaluation for participatory and sustainable planning. Londres and New York: Routledge, 2011.

LAGOS, M. Tsunamis de origen cercano a las costas de Chile. Revista de Geografía Norte Grande, 2000, Vol. 27, p. 93-102.

LAGOS, M. y CISTERNAS, M. El nuevo riesgo de tsunami: considerando el peor escenario. Scripta Nova. Revista Electrónica de Geografía y Ciencias Sociales, 2008, Vol. 12, № 270. Disponible en Internet: http://www.ub.es/geocrit/-xcol/436.htm

LANDER, E. \& CASTRO-GÓMEZ, S. La colonialidad del saber: eurocentrismo y ciencias sociales: perspectivas latinoamericanas. Buenos Aires: CLACSO, 2000.

LEFF, E. Ciencias sociales y formación ambiental. Barcelona: Editorial Gedisa, 1994.

LEFF, E. Green production: toward an environmental rationality. New York: Guilford Press, 1995.

LEFF, E. Racionalidad ambiental: la reapropiación social de la naturaleza [Environmental rationality]. Buenos Aires: Siglo XXI, 2004.

LE GOFF, J. El orden de la memoria, el tiempo como imaginario. Barcelona: Editorial Paidós, 1991.

LOSADA, R. La percepción y el análisis de riesgos. En: CASES, J. (editor). Catástrofes medioambientales: la reacción social y política. Valencia: Tirant Lo Blanch, 2010, p. 75-150.

MARDONES, M. y VIDAL, O La zonificación y evaluación de los riesgos naturales de tipo geomorfológico: un instrumento para la planificación urbana en la ciudad de Concepción. EURE, 2001, Vol. 27, $\mathrm{N}^{\circ} 81$, p. 97-122.

MARTíNEZ, M. Ciencia y arte en la metodología cualitativa. México: Trillas, 2004.

MIGNOLO, W. Delinking: The rhetoric of modernity, the logic of coloniality and the grammar of de-coloniality 1. Cultural Studies, 2007, Vol. 21, p. 449-514.

Ministerio de Desarollo Social. 2013. Casen Survey. Santiago de Chile: Ministry of Planning and Cooperation.

MONTALBA, R. Desarrollo Sostenible o Eco-Etnocidio?: El Proceso de Expansión Forestal en Territorio Mapuche-Nalche de Chile". Zaragoza: Centro de Estudios sobre la Despoblación y Desarrollo de Áreas Rurales, 2005. 
MONTEMAYOR, C. La cosmovisión de los pueblos indígenas actuales. Desacatos, 2000, № 5, p. 95-106.

MONTESPERELLI, P. Sociología de la memoria. Buenos Aires: Ediciones Nueva Visión, 2004.

PALOMINA-SCHALSCHA, M. Ejercitando el poder en tiempos neoliberales: resistencia, comodificación y emprendimiento en Alto Bío-Bío. En: BUSTOS, B.; PRIETO, M. \& BARTON, J. (editores). Ecología Política en Chile. Santiago de Chile: Editorial Universitaria, 2015, p. 213-236.

PATTON, M. Qualitative research \& evaluat ion methods. EEUU: Sage, 2002.

PEET, R.; ROBBINS, P. \& WATTS, M. (editores). Global political ecology. Londres and New York: Routledge. 2011.

PELLING, M. \& DILL, K. Disaster politics: tipping points for change in the adaptation of sociopolitical regimes. Progress in Human Geography, 2010, Vol. 34, p. 21-37.

PIAGET, J. Development explains learning. In: CAMPBELL, S. (editor). Piaget sampler. New York: John Wiley and Sonso, 1976, p. 71-88.

PÉREZ-AGUILERA, D. \& FIGUEROA-HELLAND, L. Beyond acculturation?: Political "Change", indigenous knowledges, and intercultural higher education in Mexico. Journal for Critical Education Policy Studies, 2011, Vol. 9, p. 268-296.

QUINTRIQUEO, S. \& MAHEUX, G. Exploración del conocimiento sobre la relación de parentesco como contenido educativo para un curriculum escolar intercultural en comunidades mapuche. Revista de Psicología, 2004, Vol. 13, p. 73-91.

QUIJANO, A. Colonialidad del poder, eurocentrismo y América Latina. En: LANDER, E. (editor). La colonialidad del saber: eurocentrismo y ciencias sociales. Buenos Aires: CLACSO, 2000, p. 227278.

QUILAQUEO, D. Saberes y conocimientos indígenas en la formación de profesores de educación intercultural. Educar em Revista, 2007, p. 223- 239.

QUILAQUEO, D. Interculturalidad en contexto mapuche. Cultura, Hombre, Sociedad (CUHSO), 2009, Vol. 17, p.13-21.

QUILAQUEO, D.; QUINTRIQUEO S. \& CÁRDENAS, P. Educación, Currículum e Interculturalidad. Temuco: Frasis, 2005.

RODRÍGUEZ, M. Política de desarrollo indígena y territorio mapuche: el caso del Área de DesarroIlo Indígena Lago Budi. Boletín de Geografía UMCE, 2015, №35, p. 25-42.

ROMERO TOLEDO, H. \& Romero Aravena, H. Ecología política de los desastres: vulnerabilidad, exclusión socio-territorial y erupciones volcánicas en la patagonia chilena. Magallania (Punta Arenas), 2015, Vol. 43, p. 7-26. 
RUIZ, V. \& FIGUEROA, R. Índice bibliográfico sobre peces de aguas continentales y estuarinas de Chile. Boletín Sociedad Biología de Concepción, 2006, Vol. 77, p. 73-110.

SLETTO, B.; BRYAN, J.; TORRADO, M.; HALE, C. \& BARRY, D. Territorialidad, mapeo participativo y política sobre los recursos naturales: la experiencia de América Latina. Cuadernos de Geografía, 2013, Vol. 22, p. 193-209.

SMITH, N. Uneven development: Nature, capital, and the production of space. Atenas: University of Georgia Press, 2008.

SRINIVAS, H. \& NAKAGAWAB, Y. Environmental implications for disaster preparedness: Lessons Learnt from the Indian Ocean Tsunami. Journal of Environmental Management, 2008, Vol. 89, p. 4-13.

SWYNGEDOUW, E. Globalisation or 'glocalisation'? Networks, territories and rescaling. Cambridge review of international affairs, 2004, Vol. 17, p. 25-48.

URKIDI, L. A glocal environmental movement against gold mining: Pascua-Lama in Chile. Ecological Economics, 2010, Vol. 70, p. 219-227.

UÇKLAY, A.; SAX, S.; HARBARTH, S.; BERNARD, L. \& PITTET, A. Multi-resistant infections in repatriated patients after natural disasters: lessons learned from the 2004 tsunami for hospital infection control. Journal of Hospital Infection, 2008, $\mathrm{N}^{\circ} 68$, p. 1-8.

WALKER, P. Political ecology: where is the ecology? Progress in Human Geography, 2005, Vol. 29, p. 73-82.

WALSH, C. Interculturalidad, conocimientos y decolonialidad. Signo y Pensamiento, 2005, Vol. 24, p. 39-50.

WARF, B. Advancing Human Geography at the Commencement du Siècle. The Professional Geographer, 2004, Vol. 56, p. 44-52.

WATTS, M. Now and then: the origins of political ecology and the rebirth of adaptation as a form of thought. In: PERREAULT, T.; BRIDGE, G. \& MCCARTHY, J. (editores). The Routeledge Handbook of Political Ecology. London and New York: Routledge, 2015, p. 19-50.

WILCHES-CHAUX, G. Auge, caída y levantada de Felipe Pinillo, mecánico y soldador o yo voy a correr el riesgo. Guía de LA RED para la Gestión Local del Riesgo. Lima: Red de Estudios Sociales para la Prevención de Desastres en América Latina, 1998.

WHITEHEAD, M. Cold Monsters and Ecological Leviathans: Reflections on the Relationships between States and the Environment. Geography Compass, 2008, Vol. 2, p. 414-432.

WISNER, B.; BLAIKIE, P.; CANNON, T. \& DAVIS, I. At risk: natural hazards, people's vulnerability and disasters. London and New York: Routledge. 2004. 
